\title{
Correction to: Epidermal Growth Factor Receptor (EGFR)-Tyrosine Kinase Inhibitors (TKIs) Combined with Chemotherapy Delay Brain Metastasis in Patients with EGFR-Mutant Lung Adenocarcinoma
}

\author{
Changhui $\mathrm{Li}^{1}$ - Bo Zhang ${ }^{1}$ - Jindong Guo ${ }^{2}$. Fang Hu${ }^{1} \cdot$ Wei Nie ${ }^{1} \cdot$ Xiaoxuan Zheng ${ }^{1}$ - Lixin Wang ${ }^{3}$ ' Yuqing Lou ${ }^{1}$. \\ Yinchen Shen ${ }^{1} \cdot$ Baohui Han ${ }^{1} \cdot$ Xueyan Zhang ${ }^{1}$ (i)
}

Published online: 17 July 2019

๑) Springer Nature Switzerland AG 2019

\section{Correction to: Targeted Oncology https://doi.org/10.1007/s11523-019-00649-1}

The article Epidermal Growth Factor Receptor (EGFR)-Tyrosine Kinase Inhibitors (TKIs) Combined with Chemotherapy Delay Brain Metastasis in Patients with EGFR-Mutant Lung Adenocarcinom, written by Changhui Li, Bo Zhang, Jindong Guo, Fang Hu, Wei Nie, Xiaoxuan Zheng, Lixin Wang, Yuqing Lou, Yinchen Shen, Baohui Han, Xueyan Zhang, was originally published electronically on the publisher's internet portal (currently SpringerLink) on 03 July 2019 with open access. With the author(s)' decision to step back from Open Choice, the copyright of the article changed on 17 July 2019 to () Springer Nature Switzerland AG 2019 and the article is forthwith distributed under the terms of copyright.

The original article has been corrected.

The original article can be found online at https://doi.org/10.1007/ s11523-019-00649-1.

Baohui Han

xkyyhan@gmail.com

$\triangle$ Xueyan Zhang

zxychest@163.com

1 Department of Pulmonary Medicine, Shanghai Chest

Hospital, Shanghai Jiao Tong University, Shanghai 200030,

China

2 Department of Radiation Oncology, Shanghai Chest Hospital, Shanghai Jiao Tong University, Shanghai 200030, China

3 Integrated TCM and Western Medicine Department, Shanghai Pulmonary Hospital Affiliated to Tongji University, Shanghai 200030, China 